\title{
Stability of quantum motion in regular systems: A uniform semiclassical approach
}

\author{
Wen-ge Wang, ${ }^{1,2}$ G. Casati, ${ }^{3,4,1}$ and Baowen $\mathrm{Li}^{1,5,6}$ \\ ${ }^{1}$ Department of Physics and Centre for Computational Science and Engineering, National University of Singapore, \\ 117542, Republic of Singapore \\ ${ }^{2}$ Department of Physics, Southeast University, Nanjing 210096, China \\ ${ }^{3}$ Center for Nonlinear and Complex Systems, Università degli Studi dell'Insubria, Via Valleggio 11, 22100 Como, Italy \\ ${ }^{4}$ CNR-INFM and Istituto Nazionale di Fisica Nucleare, Sezione di Milano, Italy \\ ${ }^{5}$ Laboratory of Modern Acoustics and Institute of Acoustics, Nanjing University, 210093, China
}

${ }^{6}$ NUS Graduate School for Integrative Sciences and Engineering, National University of Singapore, 117597, Republic of Singapore

(Received 27 November 2005; revised manuscript received 29 November 2006; published 5 January 2007)

\begin{abstract}
We study the stability of quantum motion of classically regular systems in the presence of small perturbations. On the basis of a uniform semiclassical theory we derive the fidelity decay which displays a quite complex behavior, from Gaussian to power law decay $t^{-\alpha}$, with $1 \leqslant \alpha \leqslant 2$. Semiclassical estimates are given for the time scales separating the different decaying regions, and numerical results are presented which confirm our theoretical predictions.
\end{abstract}

DOI: 10.1103/PhysRevE.75.016201

PACS number(s): $05.45 . \mathrm{Mt}, 03.65 . \mathrm{Sq}$

Stable manipulation of quantum states is of importance in many research fields such as in quantum information processing and in Bose-Einstein condensation. A measure of the stability of quantum motion is the so-called fidelity or quantum Loschmidt echo [1], which characterizes the stability of quantum dynamics under small perturbations of the Hamiltonian that may derive from static imperfections or from interaction with an external environment. From a more general point of view it is interesting to understand the behavior of fidelity in relation to the dynamical properties of the system.

While fidelity decay in classically chaotic systems has been extensively studied [2-11], the situation in regular systems is much less clear [5,10-19] and only for the particular case of vanishing time-average perturbations has a clear understanding been achieved [12]. Moreover, for a general perturbation and for a single initial Gaussian wave packet, a Gaussian decay has been predicted in some time interval which is not exactly specified [5]. On the other hand, numerical investigations show a much richer behavior of the fidelity decay, ranging from power law to exponential up to a Gaussian decay, depending on initial conditions, perturbation strength, and time interval [15]. In addition, a somehow unexpected regime in which the fidelity decay in regular systems is faster than in classically chaotic systems has been found in [5]. All of the above call for a theory which can account for these diverse analytical and numerical findings.

We would like to draw the reader's attention to the fact that in the general theory of dynamical systems, integrability is the exception rather than the rule and it is therefore extremely rare. However, the theory we present in this paper applies also to integrable regimes of systems with divided phase space in which both chaotic and integrable components are present and this is indeed the typical situation. Moreover, as shown in [20], for a proper operability of a quantum computer it is desirable to remain below the border for transition to quantum chaos, a situation which is likely to correspond to quasi-integrable behavior. Finally, some quantum algorithm can have a phase space representation and, in particular, the Grover algorithm can be interpreted as a simple quantum map which turns out to be a regular map [21].

The above considerations have motivated our interest in the stability of integrable motion. In this paper, we develop a uniform semiclassical approach to the fidelity decay in regular systems and we provide a unified description together with the corresponding time scales. Numerical computations confirm our analytical estimates.

Quantitatively, the fidelity for an initial state $\left|\Psi_{0}\right\rangle$ is defined as $M(t)=|m(t)|^{2}$, where

$$
m(t)=\left\langle\Psi_{0}\left|\exp (i H t / \hbar) \exp \left(-i H_{0} t / \hbar\right)\right| \Psi_{0}\right\rangle
$$

Here $H_{0}$ and $H=H_{0}+\epsilon V$ are the unperturbed and perturbed Hamiltonians, with $\epsilon$ a small quantity and $V$ a generic perturbing potential.

Consider an initial Gaussian wave packet in a $2 d$-dimensional phase space, centered at $\left(\widetilde{\mathbf{r}}_{0}, \widetilde{\mathbf{p}}_{0}\right)$,

$$
\psi_{0}\left(\mathbf{r}_{0}\right)=\left(\pi \xi^{2}\right)^{-d / 4} \exp \left[i \widetilde{\mathbf{p}}_{0} \cdot \mathbf{r}_{0} / \hbar-\left(\mathbf{r}_{0}-\widetilde{\mathbf{r}}_{0}\right)^{2} /\left(2 \xi^{2}\right)\right]
$$

For a sufficiently narrow, initial Gaussian packet, the semiclassical approximation to the fidelity amplitude is

$$
m_{\mathrm{sc}}(t) \simeq\left(\pi w_{p}^{2}\right)^{-d / 2} \int d \mathbf{p}_{0} \exp \left[\frac{i}{\hbar} \Delta S-\frac{\left(\mathbf{p}_{0}-\widetilde{\mathbf{p}}_{0}\right)^{2}}{w_{p}^{2}}\right],
$$

where $w_{p}=\hbar / \xi$ and $\Delta S$ is the action difference between the two nearby trajectories of the two systems $H$ and $H_{0}$ starting at $\left(\mathbf{p}_{0}, \widetilde{\mathbf{r}}_{0}\right)[7]$. We mention that, for not very narrow initial Gaussian packets, Eq. (3) may still hold with a redefinition of $w_{p}$ [9]. For more general initial states, the fidelity amplitude can be expressed in terms of the Wigner function of the initial state in a uniform semiclassical approach [10].

The action difference can be calculated in the first-order classical perturbation theory: 


$$
\Delta S \simeq \epsilon \int_{0}^{t} d t^{\prime} V\left[\mathbf{r}\left(t^{\prime}\right), \mathbf{p}\left(t^{\prime}\right)\right]
$$

with $V$ evaluated along one of the two trajectories. Equations (3) and (4) give quite accurate predictions even for relatively long times, much more accurate than that usually expected for a first-order perturbation treatment $[7,8]$. The reason for the unexpected accuracy is explained in [10] by making use of the shadowing theorem, the two trajectories for $\Delta S$ being in fact the so-called shadowing trajectories of the two systems with slightly different initial conditions.

Equation (3) shows that the behavior of fidelity is mainly determined by two factors: (i) $\Delta S$ as a function of $\mathbf{p}_{0}$ and $t$ and (ii) the Gaussian term, which specifies an effective window in the $\mathbf{p}_{0}$ space with size $w_{p}$. For simplicity, in what follows, we consider kicked systems with $d=1$ and set the domains of $r$ and $p$ to be $[0,2 \pi)$.

For an integrable system or a regular region of a mixed system, at least locally there exist action-angle variables $(\theta, I)$, connected to the variables $(r, p)$ by a canonical transformation. The integrand in Eq. (4) can then be written as

$$
V_{t} \equiv V[I(t), \theta(t)], \quad \text { with } \theta(t)=\theta_{0}+\nu t,
$$

$I(t) \simeq I_{0}$, and $\nu \equiv \partial H(I) / \partial I$.

The main features of $\Delta S$ as a function of $t$ and $p_{0}$ can be seen by substituting Eq. (5) into Eq. (4), replacing $\nu t$ by $\phi$ $=\nu t$, and noting the periodicity of the angle $\theta$. This gives

$$
\begin{gathered}
\Delta S \simeq \epsilon\left(U_{I} t+S_{f}\right), \quad \text { where } U_{I} \equiv \frac{1}{2 \pi} \int_{0}^{2 \pi} V(I, \theta) d \theta \\
S_{f} \equiv \frac{1}{\nu}\left[\int_{0}^{b} V\left(I, \theta_{0}+\phi\right) d \phi-b U_{I}\right] .
\end{gathered}
$$

Here $b \equiv \nu t-2 \pi n_{t}$, where $n_{t}$ is the integer part of $\nu t / 2 \pi$. Therefore, for a fixed $p_{0}, b$ is a sawtooth-type function of time oscillating between 0 and $2 \pi$ with a frequency $\nu / 2 \pi$. As a result $S_{f}$ oscillates correspondingly; hence, $\Delta S$ oscillates around its linearly increasing part $\epsilon U_{I} t$. On the other hand, for a fixed $t, U_{I}$ as a function of $p_{0}$ changes almost linearly in the neighborhood of $\tilde{p}_{0}$ while $S_{f}$ oscillates with a frequency $\widetilde{\nu}^{\prime} t / 2 \pi$, where $\nu^{\prime} \equiv \partial \nu / \partial p_{0}$. Therefore the average slope of $\Delta S$ with respect to $p_{0}$ is $\epsilon U_{I}^{\prime} t$, where $U_{I}^{\prime} \equiv \partial U_{I} / \partial p_{0}$. [We use a tilde above a quantity to indicate its value taken at the center $\left(\widetilde{r}_{0}, \widetilde{p}_{0}\right)$.]

We first study the fidelity decay for times $t<\tau_{1}$, where $\tau_{1}$ is the time scale before which the right-hand side of Eq. (3) can be calculated by a linear approximation to $\Delta S$ with respect to $p_{0}-\widetilde{p}_{0}$. This gives

$$
M_{\mathrm{sc1}}(t) \simeq \exp \left[-\frac{1}{2}\left(\sigma w_{p} \tilde{k}_{p}\right)^{2}\right], \quad \text { with } k_{p} \equiv \frac{1}{\epsilon} \frac{\partial \Delta S}{\partial p_{0}},
$$

where $\sigma=\epsilon / \hbar$ is the strength of perturbation. The explicit dependence of $k_{p}$ on time $t$ can be calculated by using Eq. (6). The leading terms give

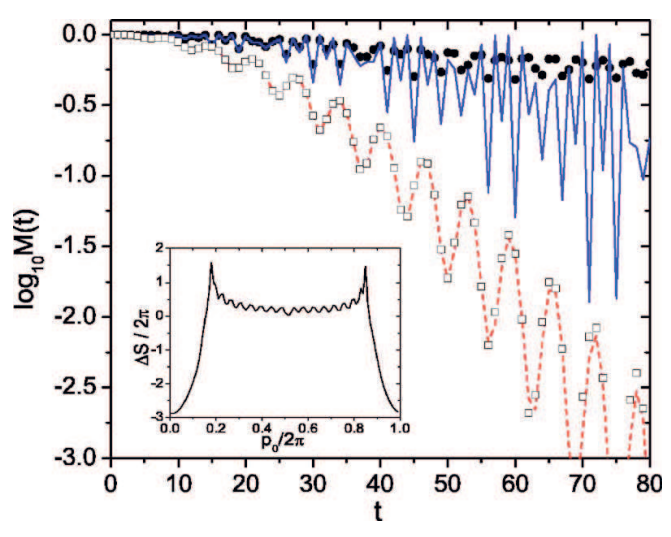

FIG. 1. (Color online) Comparison between the numerically computed fidelity decay (black circles and open squares) and the semiclassical prediction $M_{\mathrm{sc} 1}(t)$ in Eq. (8), in the kicked rotator model with $k=0.3, \sigma=1.5, N=2^{17}$, and $\xi^{2}=\hbar / 20$. Centers of the initial Gaussian packets are $\left(\widetilde{r}_{0}, \tilde{p}_{0}\right)=(1.2 \pi, 0.6 \pi)$ for the circles and $(1.2 \pi, 0.2 \pi)$ for the squares. The solid and dashed curves represent the semiclassical predictions, respectively, with $k_{p}$ evaluated numerically in the classical systems. Inset: $\Delta S / 2 \pi$ vs $p_{0} / 2 \pi$, for $r_{0}$ $=1.2 \pi$ at $t=20$. The average slope of $\Delta S$ in the monotonically increasing part is much larger than that in the central part; therefore, the fidelity decay at $\widetilde{p}_{0}=0.2 \pi$ is faster than that at $\widetilde{p}_{0}=0.6 \pi$. The oscillations of $\Delta S$ in the central region imply larger second- and higher-order terms in the expansion of $\Delta S$ around $\widetilde{p}_{0}=0.6 \pi$, hence larger deviations of the fidelity from $M_{\mathrm{sc} 1}(t)$.

$$
k_{p} \simeq\left(U_{I}^{\prime}+U_{\theta}\right) t, \quad \text { where } U_{\theta} \equiv \frac{1}{t} \frac{\partial S_{f}}{\partial p_{0}} \simeq\left(V_{t}-U_{I}\right) \frac{\nu^{\prime}}{\nu} .
$$

Since $U_{\theta}$ oscillates in time around zero, the fidelity has, on average, an initial Gaussian decay with a rate depending on initial conditions, with larger values of $\left|U_{I}^{\prime}\right|$ implying faster decay.

To test the above predictions, we consider the kicked rotator model $H=\frac{1}{2} p^{2}+k_{0} \cos r \sum_{n=0}^{\infty} \delta(t-n T)$. The quantized system has a finite Hilbert space with dimension $N$. We take $k_{0}=k N / 2 \pi$ and $T=2 \pi / N$, with $k=k_{0} T$ independent of $N$. The classical limit corresponds to $N \rightarrow \infty$. The one-period quantum evolution is given by the Floquet operator $U$ $=\exp \left[-i \hat{p}^{2} T / 2\right] \exp \left[-i k_{0} \cos (\hat{r})\right]$ and is numerically computed by the method of fast Fourier transform. Here $2 \pi / N$ serves as an effective Planck constant. The inset of Fig. 1 shows an example of $\Delta S$ vs $p_{0}$, in which the values of $\left|U_{I}^{\prime}\right|$ are large at the borders while quite small in the central, oscillating region of $p_{0}$. In agreement with our theory, under the perturbation $k \rightarrow k+\epsilon$, the fidelity for initial states lying in the two regions of $p_{0}$ has a quite different decaying rate, as seen in Fig. 1. The agreement with the theory is particularly good in the case where the oscillations of $\Delta S$ are not too strong.

The time scale $\tau_{1}$ can be estimated by the time at which the second-order term in the Taylor expansion of $\Delta S / \hbar$ is order of 1 at the point $p_{0}=\widetilde{p}_{0}+w_{p}$-i.e., $\sigma w_{p}^{2}\left|\widetilde{k}_{p p}(t)\right| \sim 1$ for $t=\tau_{1}$, where 


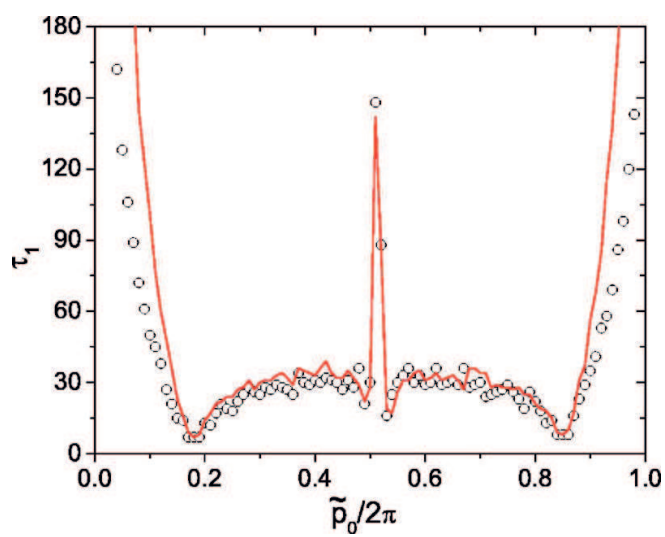

FIG. 2. (Color online) The time scale $\tau_{1}$ versus $\widetilde{p}_{0}$, for the same parameters as in Fig. 1. The circles give the values of $\tau_{1}$ calculated by the first time at which $\left|\left[M(t)-M_{\mathrm{scl}}(t)\right] / M(t)\right|>0.1$ [see Eq. (8)]. The solid curve is the semiclassical estimate given by $\sigma w_{p}^{2}\left|\widetilde{k}_{p p}\left(\tau_{1}\right)\right|=1$, with $\tilde{k}_{p p}(t)$ numerically computed in the corresponding classical system.

$$
k_{p p}(t) \equiv \frac{\partial k_{p}}{\partial p_{0}}=\frac{\left(\nu^{\prime}\right)^{2}}{\nu} \frac{\partial V}{\partial \theta} t^{2}+O(t) .
$$

A numerical check of this prediction is presented in Fig. 2. When the $t^{2}$ term dominates, $\tau_{1} \propto \sqrt{1 / \sigma w_{p}^{2}}$.

Beyond $\tau_{1}$, a direct analytical computation of the fidelity is difficult since higher- and higher-order terms in the Taylor expansion of $\Delta S$ with respect to $p_{0}-\widetilde{p}_{0}$ need to be considered. We take therefore the following approach: we divide the domain of $p_{0}$ into segments separated by points $p_{0 j}$ (with $\left.p_{00}=\tilde{p}_{0}\right)$, in such a way that $S_{f}$ completes one oscillation period within each segment. (We will use the subscript $j$ to indicate quantities taken at the point $p_{0 j}$.) It is easy to see that $\left(p_{0 j+1}-p_{0 j}\right) \nu_{j}^{\prime} t \simeq 2 \pi$ and therefore the number of segments increases linearly with time $t$. The quantity $m_{\mathrm{sc}}(t)$ in Eq. (3) can now be written as a sum of contributions $m_{j}(t)$ of different segments: namely, $m_{\mathrm{sc}}(t)=\sum_{j} m_{j}(t)$.

Let us introduce the time scale $\tau_{s}$, at which $S_{f}$ completes one oscillation within the window $W_{p}=\left(\widetilde{p}_{0}-w_{p}, \widetilde{p}_{0}+w_{p}\right)$. From Eq. (7), $\tau_{s} \approx \pi /\left(\widetilde{\nu}^{\prime} w_{p}\right)$. For $t \gg \tau_{s}$, there are many segments within $W_{p}$; hence, within each segment, the variation of the Gaussian term on the right-hand side of Eq. (3) is negligible. Therefore one may write

$$
\begin{gathered}
m_{j}(t) \simeq \frac{e^{-\left(p_{0 j}-\tilde{p}_{0}\right)^{2} / w_{p}^{2}}}{\sqrt{\pi} w_{p} \nu_{j}^{\prime} t} e^{i \Delta S_{j} / \hbar} F(t), \\
F(t)=\int_{0}^{2 \pi} d \phi \exp \left\{\frac{i \sigma}{\widetilde{\nu}} \int_{\tilde{\theta}(t)}^{\tilde{\theta}(t)+\phi} V\left(\tilde{I}, \theta^{\prime}\right) d \theta^{\prime}\right\} .
\end{gathered}
$$

Indeed for $p_{0 j}$ not far from $\widetilde{p}_{0}, F(t)$ is independent of $j$, and since $|F(t)|$ does not decay with time, we will not consider it further.

First we notice that Eq. (11) predicts a plateau in the fidelity decay when the change of $\Delta S_{j} / \hbar$ within the window $W_{p}$ is negligible. The plateau disappears when $\sigma w_{p} \widetilde{U}_{I}^{\prime} t \sim \pi$.

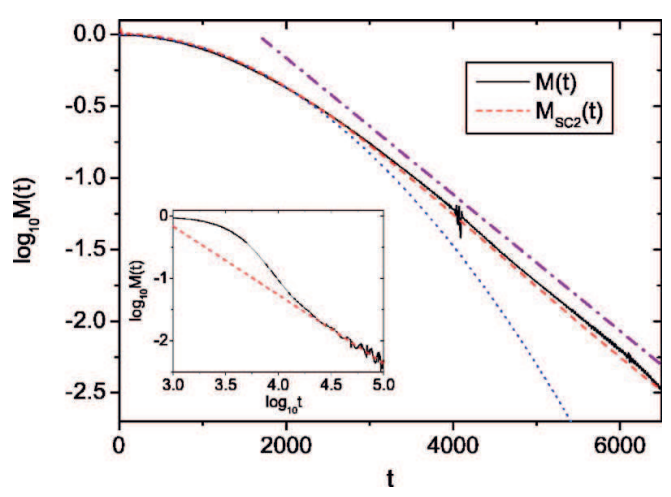

FIG. 3. (Color online) Solid curve: fidelity decay for $\sigma=0.2$, $N=2^{12}, k=0.3, \xi^{2}=\hbar / 20$, and $\left(\widetilde{r}_{0}, \widetilde{p}_{0}\right)=(1.2 \pi, 0.8 \pi)$. Dashed curve: the prediction of Eq. (14) with $c=1$ and $\tilde{U}_{I}^{\prime} \approx-0.019, \tilde{U}_{I}^{\prime \prime} \approx 0.042$ numerically computed from the classical system. Dotted curve: the Gaussian decay $e^{-\left(w_{p} \sigma \tilde{U}_{I}^{\prime} t\right)^{2} / 2}$. [For small values of $\sigma$, the difference between this decay and $M_{\mathrm{sc} 1}$ in Eq. (8) is small before $\tau_{1}$.] The dash-dotted line shows the approximate, intermediate, exponential decay. Inset: long time decay for the same case with $\widetilde{r}_{0}=0.6 \pi$ (every 500 steps shown). The dashed line gives the $1 / t^{1.1}$ decay.

If, for example, $U_{I}=0$ for the system $H_{0}$, then $U_{I} \propto \epsilon$ for the system $H$, and the plateau will end at a time proportional to $\hbar^{1 / 2} \epsilon^{-2}$ for $\xi \propto \hbar^{1 / 2}$ in agreement with the result of [12].

Due to the Gaussian term on the right-hand side of Eq. (11), the main contribution to $m_{\mathrm{sc}}(t)$ comes from $p_{0 j}$ not far from $\tilde{p}_{0}$. For these $p_{0 j}, \nu_{j}^{\prime}$ is almost a constant, as well as the value of $S_{f}$; hence, apart from a common phase, $\Delta S_{j}$ in Eq. (11) can be approximated by $\epsilon U_{I j} t$. For a sufficiently large number of segments, the sum $\sum_{j} m_{j}(t)$ can be replaced by an integral over $p_{0}$. Then, setting $q=p_{0}-\widetilde{p}_{0}$, we have

$$
m_{\mathrm{sc}}(t) \simeq \frac{F(t)}{2 \pi \sqrt{\pi} w_{p}} \int_{-\infty}^{\infty} d q e^{-q^{2} / w_{p}^{2}} e^{i \sigma t U_{I}} .
$$

Expanding $U_{I}$ to the second-order terms in $q$, we have

$$
M_{\mathrm{sc} 2}(t) \simeq \frac{2 c}{\sqrt{4+\left(w_{p}^{2} \sigma \tilde{U}_{I}^{\prime \prime} t\right)^{2}}} \exp \left[\frac{-2\left(w_{p} \sigma \tilde{U}_{I}^{\prime} t\right)^{2}}{4+\left(w_{p}^{2} \sigma \widetilde{U}_{I}^{\prime \prime} t\right)^{2}}\right]
$$

where $U_{I}^{\prime \prime} \equiv \partial U_{I}^{\prime} / \partial p_{0}$ and $c$ is a constant with $c \approx 1$ for sufficiently small $\sigma$.

From Eq. (14) it is seen that for $\left(w_{p}^{2} \sigma \widetilde{U}_{I}^{\prime \prime} t\right)^{2} \ll 4$, the fidelity has a Gaussian decay $e^{-\left(w_{p} \sigma \tilde{U}_{I}^{\prime} t\right)^{2} / 2}$, which agrees with the one given in [5] for weak perturbations. On the other hand, if $\left(w_{p}^{2} \sigma \tilde{U}_{I}^{\prime \prime} t\right)^{2} \gg 4$-i.e., for larger time $t-M_{\mathrm{sc} 2}(t)$ in Eq. (14) has a power law decay $1 / t$. In the transition region between the two decays, the fidelity may have an approximate exponential decay (see Fig. 3) which can explain the exponentiallike decay found numerically in [15].

With further increasing time $t$, higher-order terms in the Taylor expansion of $U_{I}$ will become important and will modify the $1 / t$ decay. In order to evaluate the effect of higher-order terms, we divide the interval $W_{p}$ into subintervals labeled by $l$, in such a way that inside each of them the linear approximation of $U_{I}$ can be used. Thus their width 
must be of the order $\delta q \simeq 1 / \sqrt{\left|U_{I}^{\prime \prime}\right| \sigma t}$ and their number in the region $W_{p}$ is $l_{m} \propto \sqrt{\sigma t}$. For sufficiently long time $t, l_{m} \gg 1$ and the width of each subinterval is much smaller than $W_{p}$. As a result, the Gaussian term in Eq. (13) can be regarded as constant within each subinterval. Then, the integral (13) reduces to $m_{\mathrm{sc}}(t) \simeq \Sigma_{l} m_{l}(t)=\Sigma_{l} c_{l} e^{i \phi_{l}(t)} / \sigma t$, with some coefficients $c_{l}$ and phases $\phi_{l}(t)$. The detailed behavior of this sum depends on the properties of the function $U_{I}$. However, one can give estimates in some limiting cases: (i) Since $\left|\Sigma_{l} c_{l} e^{i \phi_{l}(t)}\right| \leqslant \Sigma_{l}\left|c_{l}\right| \propto(\sigma t)^{1 / 2}$, the slowest decay is $1 / \sqrt{\sigma t}$. (ii) The fastest decay is obtained when $\left|\Sigma_{l} c_{l} e^{i \phi_{l}(t)}\right|$ is a constant (which may happen due to mutual cancellation of phases $\phi_{l}$ ). In this case, $m_{\mathrm{sc}} \sim 1 / \sigma t$. (iii) In the case of random phases $\phi_{l}$, one has $\left|\Sigma_{l} c_{l} e^{i \phi_{l}(t)}\right| \propto(\sigma t)^{1 / 4}$, then $m_{\mathrm{sc}} \sim 1 /(\sigma t)^{3 / 4}$, which coincides with the result given in Ref. [13] for averaged fidelity. (For an analysis of Gaussian and power law decay of fidelity, which is based on statistics of action difference, see Ref. $[10(\mathrm{c})])$.

Therefore, in general, the decay of $M(t)$ has the power law dependence $(\epsilon t / \hbar)^{-\alpha}$ with $1 \leqslant \alpha \leqslant 2$. This is in agreement with numerical results in [15], as well as with our extensive numerical simulations (see, e.g., Fig. 4 and the inset of Fig. 3). The cases of $\alpha \simeq 1$ or 2 have been found to be quite rare in our simulations.

It is important to remark that, contrary to chaotic systems for which the decay rate depends on the strength but not on the shape of the perturbation, integrable systems lack of such generic behavior. This is typical in the general theory of dynamical systems, and it is due to the peculiarity of integrability. In the present case the numerical value of $\alpha$ depends on the particular shape of $U_{I}$.

Our approach can also explain an interesting feature of fidelity decay in regular systems observed numerically in [15], that is the fact that, in some cases, the decay rate may decrease with increasing the perturbation strength $\sigma$. First we note that Eq. (14) is valid only when $\left|\Delta S_{j+1}-\Delta S_{j}\right| / \hbar$ is small compared with $\pi$, since it is obtained by replacing the sum $\sum_{j} m_{j}(t)$ by an integral over $p_{0}$. Since $U_{I}^{\prime}$ is given by $\left(\Delta S_{j+1}\right.$ $\left.-\Delta S_{j}\right) /\left(p_{0 j+1}-p_{0 j}\right) \epsilon t$, then $\left|\Delta S_{j+1}-\Delta S_{j}\right| / \hbar \simeq 2 \pi \sigma\left|U_{I j}^{\prime} / \nu_{j}^{\prime}\right|$, which may exceed $\pi$ for sufficiently large $\sigma$. In this case, we can replace $\Delta S_{j} / \hbar$ by $\psi_{j} \equiv \Delta S_{j} / \hbar-2 \pi m j$, where $m$ is an integer such that $\left|\psi_{j+1}-\psi_{j}\right| \leqslant \pi$. Then using the fact that, for $p_{0 j}$ close to $\widetilde{p}_{0},\left(p_{0 j}-\widetilde{p}_{0}\right) \widetilde{\nu}^{\prime} t \simeq 2 \pi j$, we find that the term $\sigma \widetilde{U}_{I}^{\prime}$ in Eq. (14) can be replaced by $\beta \equiv\left|\sigma \widetilde{U}_{I}^{\prime}-m \widetilde{\nu}^{\prime}\right|$. With increasing $\sigma$, the value of $\beta$, which gives the decay rate, oscillates between 0 and $\left|\widetilde{\nu}^{\prime}\right| / 2$, with a period $\left|\widetilde{\nu}^{\prime} / \widetilde{U}_{I}^{\prime}\right|$. The results of Fig. 4 nicely confirm the above analysis.

Finally a word of comment on the comparison of fidelity

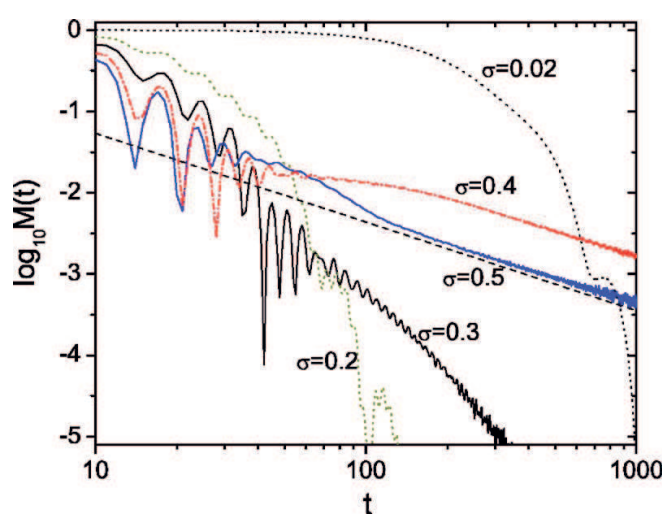

FIG. 4. (Color online) Fidelity decay for several values of $\sigma$ with $k=0.3, N=2^{12}, \xi^{2}=\hbar / 20$, and $\left(\widetilde{r}_{0}, \widetilde{p}_{0}\right)=(1.2 \pi, 0.28 \pi)$. For $t$ around 100 , the rate of fidelity decay increases for $0<\sigma<0.2$, then decreases for $0.2<\sigma<0.4$, and then increases again. This is in agreement with the semiclassical prediction that the decay rate $\beta$ oscillates with a period $\left|\widetilde{\nu}^{\prime} / \widetilde{U}_{I}^{\prime}\right| \approx 0.4$ (see text). The values $\tilde{U}_{I}^{\prime}$ $\approx 1.85$ and $\widetilde{\nu}^{\prime} \approx 0.76$ are obtained from the classical motion. Notice that the transition time from Gaussian to power law decay of fidelity is proportional to $1 / \sigma$ (see text). Here the power law decay is visible for $\sigma=0.4$ and 0.5 (the dashed straight line gives the $1 / t^{1.09}$ decay).

decay in classically regular and chaotic systems. As shown in [5], in a sufficiently weak perturbation regime $\sigma<\sigma_{c} \propto \sqrt{\hbar}$, the Gaussian decay $e^{-\left(w_{p} \sigma \widetilde{U}_{I}^{\prime} t\right)^{2} / 2}$ in the regular case can be faster than the Fermi-golden-rule decay in the chaotic case. This is not surprising as it may look at first sight, since in this regime, $w_{p} \sigma_{c} \propto \hbar$ can be quite small and the fidelity may remain close to 1 for a time comparable to the Heisenberg time $\sim 1 / \hbar$, which can be quite long. Moreover, the above Gaussian decay in the regular case is followed by a power law decay, which is slower than the exponential decay in the chaotic case.

We thank T. Prosen for useful discussions. This work was supported in part by the Academic Research Fund of the National University of Singapore and the Temasek Young Investigator Award (B.L.) of DSTA Singapore under Project Agreement No. POD0410553. Support was also given by the EC RTN Contract No. HPRN-CT-2000-0156, the NSA and ARDA under ARO Contract No. DAAD19-02-1-0086, the project EDIQIP of the IST-FET programme of the EC, the PRIN-2002 "Fault tolerance, control and stability in quantum information processing," and Natural Science Foundation of China Grant No. 10275011.
[1] A. Peres, Phys. Rev. A 30, 1610 (1984)

[2] R. A. Jalabert and H. M. Pastawski, Phys. Rev. Lett. 86, 2490 (2001).

[3] Ph. Jacquod, P. G. Silvestrov, and C. W. J. Beenakker, Phys. Rev. E 64, 055203(R) (2001); P. G. Silvestrov, J. Tworzydło, and C. W. J. Beenakker, Phys. Rev. E 67, 025204(R) (2003).
[4] N. R. Cerruti and S. Tomsovic, Phys. Rev. Lett. 88, 054103 (2002); J. Phys. A 36, 3451 (2003).

[5] T. Prosen and M. Žnidarič, J. Phys. A 35, 1455 (2002).

[6] G. Benenti and G. Casati, Phys. Rev. E 65, 066205 (2002).

[7] J. Vaníček and E. J. Heller, Phys. Rev. E 68, 056208 (2003).

[8] W.-G. Wang, G. Casati, and B. Li, Phys. Rev. E 69, 025201(R) 
(2004); W.-G. Wang, G. Casati, B. Li, and T. Prosen, ibid. 71, 037202 (2005).

[9] W.-G. Wang and B. Li, Phys. Rev. E 71, 066203 (2005).

[10] J. Vaníček, Phys. Rev. E 70, 055201(R) (2004); 73, 046204 (2006); e-print quant-ph/0410205.

[11] T. Gorin, T. Prosen, T. H. Seligman, and M. Žnidarič, Phys. Rep. 435, 33 (2006).

[12] T. Prosen and M. Žnidarič, New J. Phys. 5, 109 (2003).

[13] Ph. Jacquod, I. Adagideli, and C. W. J. Beenakker, Europhys. Lett. 61, 729 (2003).

[14] R. Sankaranarayanan and A. Lakshminarayan, Phys. Rev. E 68, 036216 (2003).

[15] Y. S. Weinstein and C. S. Hellberg, Phys. Rev. E 71, 016209
(2005).

[16] M. Combescure, J. Phys. A 38, 2635 (2005); J. Math. Phys. 47, 032102 (2006); M. Combescure and D. Robert, e-print quant-ph/0510151.

[17] F. Haug, M. Bienert, W. P. Schleich, T. H. Seligman, and M. G. Raizen, Phys. Rev. A 71, 043803 (2005).

[18] Jie Liu, Wenge Wang, Chuanwei Zhang, Qian Niu, and Baowen Li, Phys. Rev. A 72, 063623 (2005).

[19] S. Wimberger and A. Buchleitner, J. Phys. B 39, L145 (2006).

[20] G. Benenti, G. Casati, and D. L. Shepelyansky, Eur. Phys. J. D 17, 265 (2001).

[21] C. Miquel, J. P. Paz, and M. Saraceno, Phys. Rev. A 65, 062309 (2002). 